\title{
Robust Adaptive Control of Fuzzy Nonlinear System via Variable Structure Control Approach
}

\author{
X. Z. Zhang ${ }^{1,2}$ \\ ${ }^{1}$ The CIC of Hunan Institute of Engineering, Xiangtan 411104, China \\ ${ }^{2}$ School of Electrical and Information Engineering, Hunan University, \\ Changsha 410082, China
}

\begin{abstract}
This paper proposed a novel fuzzy Variable Structure Controller (VSC) design for uncertain T-S fuzzy system, while neither assuming the control matrices of each local $T$-S model to be same nor knowing the priori information of the upper norm-bounds of uncertainties. The existence condition of desired sliding surfaces and the asymptotical stability of the reduced-order equivalent sliding-mode dynamics are firstly derived by solving a set of linear matrix inequalities (LMIs). After the establishment of the local $T$-S model, the upper norm-bounds of uncertainties and modeling errors between the original and local systems are estimated. Then, the designed VSC controller composes of a state-feedback control term and an adaptive switching-feedback control term, which are achieved based on the Lyapunov function method. Finally, a numerical example is illustrated in order to show the effectiveness of the proposed methods.
\end{abstract}

Keywords: T-S fuzzy system, robust stability, variable structure control, uncertain nonlinear systems

\section{Introduction}

It has been shown that $\mathrm{T}-\mathrm{S}$ fuzzy model is a powerful and efficient tool to handle complex nonlinear system, and be employed in most model-based fuzzy analysis approaches [1, 2]. Since the Parallel Distributed compensation (PDC) approach is formulated by Tanaka and Wang [3], the stability analysis and stabilized controller design for T-S fuzzy systems become achievable and have been applied in a wide range of areas such as electrical/mechanical systems [4, 5], process control [6], robot [7] and time-delayed systems [8]. A lot of research outcomes on this field have been appeared in literatures [4-8]. Practically, a real nonlinear system may contain various kinds of uncertainties such as parameter variations, modeling errors and external disturbances, etc. In this case, the PDCbased controllers [3] may perform well no longer. Then, the robustly stabilized control to uncertainties of nonlinear fuzzy systems is required. Based on the T-S fuzzy model, Wu studied the robust $\mathrm{H}_{2}$ fuzzy observer-based control problem for discrete-time nonlinear systems with parametric uncertainties [9]. Lin, et.al, investigated the mixed $\mathrm{H}_{2} / \mathrm{H}_{\infty}$ controller design for uncertain $\mathrm{T}$-S fuzzy systems with discrete time-varying delay [10].

It is well known that the Variable Structure Control (VSC) is an effective means to design robust controllers for nonlinear systems with uncertainties bounded by known scalar-valued functions. Recently, the VSC has been successfully applied into solving the stabilization problems for uncertain fuzzy systems $[11,12]$. Specially, under the VSC control the state trajectories would enter into the pre-designed sliding-mode motion within finite time and after that keep staying on it; thus, the system dynamics is not sensitive to parameter variations and external disturbances any more [9, 13]. Basically, the feedback 
gains of the VSC control are often determined by the feasible solution of a set of Linear Matrix Inequalities (LMI) [14]. Choi presented a robust stabilization of uncertain fuzzy systems using VSC system approach [15]. Zhang and Wang proposed a mixed $\mathrm{VSC}-\mathrm{H}_{\infty}$ controller for time-delay system with unmatched uncertainties [16].

Although many researchers have proposed a variety of T-S fuzzy VSC-based methods [4, 7-13, 15-17], by far there still two problems yet remained to be well-solved: (1) the suitable relaxation on the assumption that all the control matrices of the nominal sub-systems' models are identical; It is practically difficult to satisfy this assumption. In practice, this assumption is very strict and insufficient to model various uncertainties/nonlinearities in most of actual systems such as nonlinear stirred tank reactor, fourth-order cart-pole system, and active queue management in TCP networks and two-link robot manipulator; (2) the reasonable assumption that the system uncertainties/perturbations are unknown but norm-bounded. For the VSC-based methods, if uncertainties are known, it's easy to choose proper switching gains, which are bigger than the upper norm-bounds of uncertainties, to ensure the reachability of sliding-mode motion. Unfortunately, the information of the upper bound of uncertainties/perturbations may not easily be obtained in practice. Therefore, it's supposed to adopt parameter identification or adaptive control approach to estimate the bounds of uncertainties on-line [18].

Motivated by the above discussion, it is meaningful to design fuzzy variable structure controller in this paper such that the closed-loop system is asymptotically stable without (1) assuming the control matrices $B_{i}$ of each local linear model to be same; and (2) knowing the priori information of the upper norm-bounds of uncertainties. The existence condition of linear sliding surfaces and the asymptotical stability of the reduced-order equivalent sliding-mode dynamics are firstly derived by the LMI optimization technique. After the establishment of the local T-S model, the upper norm-bounds of uncertainties and modeling errors between the original and local systems are estimated. Then, the designed VSC controller composes of a state-feedback control term and an adaptive switching-feedback control term, which are achieved based on the Lyapunov function method. As a result, the aforementioned problems are well solved in the proposed scheme. Finally, a numerical example is illustrated in order to show the effectiveness of the proposed methods.

\section{Problem Formulation and Preliminaries}

As stated in Introduction, T-S fuzzy models can provide an effective representation of complex nonlinear systems in terms of fuzzy sets and fuzzy reasoning applied to a set of linear input-output sub-models. Hence, in this work, a class of nonlinear systems is represented by a T-S model. As in [3], the T-S fuzzy system with uncertainties is described by fuzzy IF-THEN rules, which locally represent linear input-output relations of nonlinear systems. The $i$ th rule of the fuzzy model is formulated in the following equation:

$$
\begin{aligned}
& \text { Plant rule } \mathrm{R}_{p}^{i} \text { : IF } \quad z_{1}(t) \text { is } M_{1}^{i} \text { and, } \ldots \text {, and } z_{\mathrm{n}}(t) \text { is } M_{n}^{i} \\
& \text { THEN } \quad \dot{x}(t)=A_{i} x(t)+B_{i} u(t)
\end{aligned}
$$

where $M_{j}^{i}$ is the fuzzy set, $z(t)=\left[z_{1}, \cdots, z_{\mathrm{n}}\right]$ is the premise variable vector, $i=1,2, \cdots, r$ is the number of rules of this T-S fuzzy model. $\boldsymbol{x}(t) \in \mathfrak{R}^{n}$ is the state vector, $\boldsymbol{u}(t) \in \mathfrak{R}^{m}$ is the control input vector. $\boldsymbol{A}_{i}, \boldsymbol{B}_{i}$ are known real constant matrices with appropriate dimensions. 
The overall fuzzy model achieved by fuzzy blending of each individual plant rule is represented as follows:

$$
\dot{x}(t)=\sum_{i=1}^{r} h_{i}(z)\left[A_{i} x(t)+B_{i} u(t)\right]
$$

where $h_{i}(z(t))=\frac{w_{i}(x(t))}{\sum_{j=1}^{r} w_{i}(x(t))}, \quad w_{i}(x(t))=\prod_{j=1}^{r} M_{j}^{i}\left(z_{j}(t)\right)$, in which $M_{j}^{i}\left(z_{j}(t)\right)$ is the grade of the membership of $z_{j}(t) \theta_{j}(t)$ in $M_{j}^{i}$. According to the theory of fuzzy sets, we have $M_{j}^{i}\left(z_{j}(t)\right) \geq 0, \sum_{i=1}^{r} M_{j}^{i}\left(z_{j}(t)\right) \geq 0$, and this implies that $h_{i}(z(t)) \geq 0$ and $\sum_{i=1}^{r} h_{i}(z(t))=1$.

In practical applications, the system (2) usually exists the parameters variation and the external disturbances. After considering the perturbation, the global fuzzy model of system (2) can be rewritten as

$$
\dot{x}(t)=\sum_{i=1}^{r} h_{i}(z)\left[A_{i}+\Delta A_{i}(t)\right] x(t)+\sum_{i=1}^{r} h_{i}(z)\left[B_{i}+\Delta B_{i}(t)\right] u(t)+\sum_{i=1}^{r} h_{i}(z) d_{i}(t, x)
$$

where $\Delta \boldsymbol{A}_{i}(t), \Delta \boldsymbol{B}_{i}$ are unknown time-varying matrices representing parameter uncertainties, and $d_{i}(t, x)$ denotes the external disturbance. In this work, the following assumptions are introduced.

Assumption 1. It is assumed that the matrices doesn't satisfy $\boldsymbol{B}_{1}=\boldsymbol{B}_{2}=\cdots=\boldsymbol{B}_{r}$ $(i=1,2, \cdots, r)$. Moreover, the nominal matrix $\boldsymbol{B} \in \mathfrak{R}^{n \times m}$ with full-column rank is defined as $B=\frac{1}{r} \sum_{i=1}^{r} B_{i}$.

Assumption 2. The uncertain matrice $\Delta \boldsymbol{A}_{i}(t), \Delta \boldsymbol{B}_{i}$ and $d_{i}(t, x)$ are assumed to be matched, i.e. there exist certain functions $D(t), E(t)$ and $F(t)$ such that $\Delta A_{i}(t)=B_{i} D(t), \Delta B_{i}(t)=B_{i} E(t), d_{i}(t, x)=B_{i} F(t)$ hold. Let the time-varying function $g(t, \boldsymbol{x}, u)=D(t) x(t)+E(t) u(t)+F(t)$, then $g(t, \boldsymbol{x}, u)$ contains all the perturbation in system (3).

Assumption 3. The perturbation $g(t, x, u)$ is assumed to be norm-bounded, that is $\|g(t, \boldsymbol{x}, u)\| \leq \sum_{k=0}^{N-1} \gamma_{k}\|x\|^{k}$, and the upper bound is estimated as $\hat{g}(t, \boldsymbol{x}, u)$, where $\gamma_{0}, \cdots, \gamma_{N-1}$ are unknown coefficients, $N$ is a positive integer.

Under the Assumptions 1 4, the system (3) can be rewritten as

$$
\dot{x}(t)=\sum_{i=1}^{r} h_{i}(z) A_{i} x(t)+[B+\bar{B} \bar{H}(h(z)) \bar{I}][u+g(t, x, u)]
$$

where

$$
h(z)=\left[h\left(z_{1}\right), \cdots, h\left(z_{r}\right)\right]^{T} \quad, \quad \bar{B}=\frac{1}{2}\left[\left(B-B_{1}\right), \cdots,\left(B-B_{r}\right)\right]
$$

$\bar{H}=\operatorname{diag}\left[\left(1-2 h_{1}\right) I, \cdots,\left(1-2 h_{r}\right) I\right], \bar{I}=[I, \cdots, I]^{T}$, and $\bar{H}^{T} \bar{H} \leq 1$.

Before proceeding, some standard concepts and lemma are given as following, which are useful for the development of our result.

Lemma 1 Let $a, b$ be arbitrary vectors with proper dimensions, $W$ is a positive matrix, then the inequality $2 a^{T} b \leq a^{T} W a+b^{T} W^{-1} b$ hold. 
Lemma 2 Let $A, B$ be arbitrary matrices with proper dimensions, if the inverse matrix of $(I+A B)$ exists, then we have $(I+A B)^{-1}=I-A(I+B A)^{-1} B$.

Lemma 3 (Schur's Complement [14]): Given the matrix inequality $\left[\begin{array}{ll}S_{11} & S_{12} \\ S_{12}^{T} & S_{22}\end{array}\right]<0$, where $S_{11}, S_{22}$ are positive symmetrical matrices, it's equal to each of the following inequalities:

(i) $S_{11}<0, S_{22}-S_{12}^{T} S_{11}^{-1} S_{12}<0$, (ii) $S_{22}<0, S_{11}-S_{12} S_{22}^{-1} S_{12}^{T}<0$.

\section{Controller Design}

The objective of this work is to design a VSC law such that the desired control performance for the resulting closed-loop system is obtained despite of parameter uncertainties and unmatched external disturbance. In this section, a VSC law is synthesized such that the closed-loop systems are robustly asymptotically stable. It is also proven that the reachability of the specified switching (sliding) surface $S(t)=0$ can be ensured by the proposed VSC law. Thus, it is concluded that the synthesized VSC law can guarantee the state trajectories of uncertain systems (4) to be driven onto the sliding surface, and asymptotically tend to zero along the specified sliding surface.

\subsection{Design of the Sliding Function and Stability Analysis of the Sliding Motion}

Essentially, a VSC design is composed of two phases: hyper-plane design and controller design [9]. There are various methods for designing hyper-plane, however in this paper the switching surface is defined as

$$
S=C x
$$

where $C \in R^{m \times n}$ is the coefficient. It must be pointed out that $C$ should be properly chosen to assure the existence of the equivalent control of sliding-mode [15], i.e., the matrix $C[B+\bar{B} \bar{H}(z) \bar{I}]$ is nonsingular. This means that $S(t, x)$ varies finitely. Once the system (3) enters the sliding surface $S(t, x)=0$, the asymmetrical stability should be assured.

In this subsection, we analyze the dynamic performance of the system described by (4), and derives some sufficient conditions for the asymptotically stability of the sliding dynamics via LMI method. The following theorem shows that system (4) with the switching surface (5) is robustly stable.

Theorem 1: Consider the fuzzy uncertain systems (4) with Assumptions (1)-(4). The switching function is given by (5). If there are feasible solutions $(Q, \alpha, \beta, \mu)$ such that the LMIs shown in (6), (7) and (8) hold, the proper sliding-mode coefficient $C$ exists and $C=\left(B^{T} Q^{-1} B\right)^{-1} B^{T} Q^{-1}$.

$$
\begin{aligned}
& {\left[\begin{array}{ccc}
A_{i} Q+Q A_{i}^{T} & * & * \\
\mu \bar{B}^{T} & -I & * \\
A_{i} Q & \eta \bar{B} & -I
\end{array}\right]<0, \forall i} \\
& {\left[\begin{array}{cc}
Q & I \\
* & \alpha I
\end{array}\right]>0, \quad\left[\begin{array}{cc}
Q & I \\
* & \beta^{-1} I
\end{array}\right]<0} \\
& {\left[\begin{array}{cccc}
2 \kappa \mu^{2} & \alpha & \beta & \\
* & r^{-1} \boldsymbol{I} & 0 & \\
* & * & r^{-1} \boldsymbol{I}
\end{array}\right]>0}
\end{aligned}
$$


where the invertible matrix $Q \in R^{n \times n}, \alpha, \beta$ and $\mu \in R$ are decisive variables, $\kappa^{2}$ is the minimal eigen-value of the matrix $B^{T} B$, that is $\kappa=\sqrt{\lambda_{\min }\left(B^{T} B\right)}$. The notation “*” represents the transposition of the corresponding elements of the matrix.

Proof : First, the existence of the coefficient $C$ is proved. According to Schur's complement, the LMIs (Linear Matrix Inequalities) (6), (7) and (8), it's easy to prove that the following inequalities hold

$$
\alpha>0, \beta>0, \mu>0, \bar{B}^{T} \bar{B}<\eta^{-2} I
$$

Let $G=B^{T} X, C=(G B)^{-1} G Q$. According to (8), we have $\bar{I} \cdot \bar{I}^{T} \leq\|\bar{I}\|^{2} I=r I$. With these, it can be derived that

$$
C \bar{B} \bar{H}(z) \bar{I} \cdot \bar{I}^{T} \bar{H}^{T}(z) \bar{B}^{T} C^{T} \leq \frac{r}{\mu^{2}} C \bar{B} \bar{B}^{T} C^{T} \leq \frac{r}{\mu^{2}} C C^{T}
$$

By the Schur's complement formula, we can see that (6) and (9) imply

$$
0<\beta^{-1} I<Q<\alpha I, 0<\alpha^{-1} I<Q^{-1}<\beta I
$$

And this leads to

$$
\frac{r}{\mu^{2}} C C^{T}=\frac{r}{\mu^{2}}(G B)^{-1} B^{T} Q\left[(G B)^{-1} B^{T} Q\right]^{T}<\frac{r \alpha \beta}{\kappa^{2} \mu^{2}} I
$$

Thus, the following inequality is easily derived

$$
C \bar{B} \bar{H}(z) \bar{I} \cdot \bar{I}^{T} \bar{H}^{T}(z) \bar{B}^{T} C^{T} \leq \frac{r}{2 \kappa^{2} \mu^{2}}\left(d_{1}^{2}+d_{2}^{2}\right) \boldsymbol{I}
$$

According to (13), it is obvious that the achieved matrix $C \bar{B} \bar{H}(z) \bar{I}+I$ is nonsingular. That is to say, if the coefficient is chosen as $C=(G B)^{-1} B^{T} Q$, both the sliding surface and the equivalent control exist. With the chosen sliding surface, once the $n$-order system enters the $m$-order sliding surface $S=C x=0$, the system dynamic of (4) is equivalent to the $(n-m)$-order sliding motion, and the system states will asymptotically converge to zero with proper switching gains. In the following, we will derive the equivalent control and the sliding mode as well as the stability analysis.

Now, the linear transformation $T$ is carried on the states $x$ to separate the $m$-th sliding-mode states and the reduced $(n-m)$ order state

$$
T=\left[\begin{array}{l}
T_{1} \\
T_{2}
\end{array}\right]=\left[\begin{array}{c}
\left(K^{T} Q^{-1} K\right)^{-1} K^{T} \\
(G B)^{-1} G
\end{array}\right]
$$

where $T_{1} \in R^{(n-m) \times n}, T_{2} \in R^{m \times n}, K \in R^{n \times(n-m)}$ is a coefficient matrix with full rank and should be properly selected such that $B^{T} K=0, K^{T} K=I$ satisfies. We have

$$
z=T x=\left[\begin{array}{ll}
z_{1} & z_{2}
\end{array}\right]^{\mathrm{T}}
$$

In (15), it's obvious that $z_{2}=(G B)^{-1} G x=C x=S$ is the sliding-mode state and $z_{1}$ is the reduced-order state. It's easy to obtain the inverse transformation $T^{-1}=\left[\begin{array}{ll}Q^{-1} K & B\end{array}\right]$.

By the above transformation, we can obtain

$$
\begin{aligned}
\dot{z} & =T \cdot \dot{x}=\sum_{i=1}^{r} h_{i}(z)\left\lceil\left[\begin{array}{l}
T_{1} \\
T_{2}
\end{array}\right] A_{i} \cdot\left[\begin{array}{ll}
Q^{-1} K & B
\end{array}\right] \cdot T x+\left[\begin{array}{l}
T_{1} \\
T_{2}
\end{array}\right] \cdot[B+\bar{B} \bar{H}(z) \bar{I}] \cdot[u+g(t, x, u)]\right. \\
& =\sum_{i=1}^{r} h_{i}(z)\left[\begin{array}{ll}
T_{1} A_{i} Q^{-1} K & T_{1} A_{i} B \\
T_{2} A_{i} Q^{-1} K & T_{2} A_{i} B
\end{array}\right]\left\lceil\left[\begin{array}{l}
z_{1} \\
z_{2}
\end{array}\right]+\left[\begin{array}{l}
\left.T_{1} B+T_{1} \bar{B} \bar{H}(z) \bar{I}\right\rceil \\
T_{2} B+T_{2} \bar{B} \bar{H}(z) \bar{I}
\end{array}\right] \cdot[u+g(t, x, u)]\right.
\end{aligned}
$$


In (16), it's not difficult to prove that $T_{1} B=\left(K^{T} Q^{-1} K\right)^{-1} K^{T} B=0$ and $T_{2} B=I$. According to the sliding mode theory, let $\dot{S}=S=0$, the following equivalent control can be derived

$$
u_{e q}(t)=-g(t, x, u)-\sum_{i=1}^{r} h_{i}(z)[I+C \bar{B} \bar{H}(z) \bar{I}]^{-1} C A_{i} x
$$

Substitute $u_{e q}(t)$ in (17) into (16), the reduced-order sliding motion in the switching surface can be obtained as

$$
\dot{z}_{1}=\sum_{i=1}^{r} h_{i}(z)\left(K^{T} Q^{-1} K\right)^{-1} K^{T}\left\{I-\bar{B} \bar{H}(z) \bar{I}[I+C \bar{B} \bar{H}(z) \bar{I}]^{-1} C\right\} A_{i} Q^{-1} K z_{1}
$$

To analyze the stability of the sliding-mode dynamics (8), we consider the fuzzy uncertain system (4) with LMIs in (6), (7), (8) and choose the Lyapunov functional candidate $V=z_{1}^{T} P z_{1}$, where $P=K^{T} Q^{-1} K$ is a positive matrix.

By differentiating the function $V$, we obtain the differential along the trajectories as

$$
\dot{V}_{1}=\sum_{i=1}^{r} h_{i}(z) z_{1}^{T}\left\{K^{T}\left[I-\bar{B} \bar{H}(z) \bar{I}[I+C \bar{B} \bar{H}(z) \bar{I}]^{-1} C\right] A_{i} Q^{-1} K+(*)\right\} z_{1}
$$

According to Lemma 1, it's easy to prove that $I-\bar{B} \bar{H}(z) \bar{I}[I+C \bar{B} \bar{H}(z) \bar{I}]^{-1} C$ $=I-\bar{B}[I+\bar{H}(z) \overline{I C} \bar{B}]^{-1} \bar{H} \overline{I C}$, by substituting this equality into (19), we have

$$
\dot{V}_{1}=\sum_{i=1}^{r} h_{i}(z) w^{T}\left\{\left(A_{i} Q+*\right)-\left(\bar{B}[I+\bar{H}(z) \overline{I C} \bar{B}]^{-1} \bar{H}(z) \overline{I C} A_{i} Q+*\right)\right\} w
$$

where $w=K z_{1} \in R^{n \times 1}$ is defined as new states. By now, the stability of the states $x$ in the switching surface is equivalent to the stability of $w$.

According to the inequalities (9), (11) and $\bar{I} \cdot \bar{I}^{T} \leq r I$, we have

$$
\begin{aligned}
& \bar{H}(z) \overline{I C} C^{T} \bar{I}^{T} \bar{H}^{T}(z) \leq \mu^{2} I \\
& \bar{B}^{T} \bar{B}<\mu^{-2} I
\end{aligned}
$$

Let the vector $p_{i}=-[I+\bar{H}(z) \overline{I C} \bar{B}]^{-1} \bar{H}(z) \overline{I C} A_{i} Q v$, where $v \in R^{(n-m) \times n}$ is an arbitrary vector. Then $p_{i}$ can be rewritten as

$$
p_{i}=-\bar{H}(z) \bar{I} C\left[A_{i} Q v+\bar{B} p_{i}\right]
$$

This equality implies that

$$
\begin{gathered}
p_{i}^{T} p_{i} \leq \mu^{2}\left[A_{i} Q v+\bar{B} p_{i}\right]^{T}\left[A_{i} Q v+\bar{B} p_{i}\right] \\
-2 v^{T} \bar{B}[I+\overline{H I C} \bar{B}]^{-1} \overline{H I C} A_{i} Q v=2 v^{T} \bar{B} p_{i} \leq v^{T} Q A_{i}^{T} A_{i} Q v+2 v^{T}\left[I+Q A_{i}^{T}\right] \bar{B} p_{i}-\mu^{-2} p_{i}^{T} \Gamma p_{i}
\end{gathered}
$$

where $\Gamma=I-\mu^{2} \bar{B}^{T} \bar{B}$ and $\Gamma>0$. For any vector $v$, by Lemma 1, let $a^{T}=v^{T}\left[I+Q A_{i}^{T}\right] \bar{B}, b=p_{i}, W=\mu^{2} \Gamma^{-1}$, we have

$$
2 v^{T}\left[\bar{B}+Q A_{i}^{T} \bar{B}\right] p_{i} \leq \mu^{-2} p_{i}^{T} \Gamma p_{i}+\mu^{2} z^{T}\left[I+Q A_{i}^{T}\right] \bar{B} \cdot \Gamma^{-1} \cdot\left[\bar{B}+Q A_{i}^{T} \bar{B}\right]^{T} z
$$


According to the inequalities (25), (26), we have

$$
A_{i} Q+Q A_{i}^{T}+Q A_{i}^{T} A_{i} Q+\mu^{2}\left[\bar{B}+Q A_{i}^{T} \bar{B}\right] \Gamma^{-1}\left[\bar{B}+Q A_{i}^{T} \bar{B}\right]^{T}<0
$$

By Schur's complement, the inequality (27) is equivalent to the LMI shown in (28)

$$
\left[\begin{array}{ccc}
A_{i} Q+Q A_{i}^{T} & * & * \\
\mu \bar{B}^{T} & -I & * \\
A_{i} Q & \mu \bar{B} & -I
\end{array}\right]<0 \quad, \quad \forall i
$$

This means that the sliding surface (5) and the equivalent control (17) for the fuzzy system (4) with Assumption 1-3 exist and the sliding motion is asymptotically stable.

\subsection{Design of Controller and Adaptive Laws}

As the last step of design procedure, we will further design the VSC controller ensures the reachability of the specified switching surface. The adaptive VSC controller is represented in a set of fuzzy rules as following

Controller rule $\mathrm{R}^{i}$ : IF $\quad z_{1}(t)$ is $M_{1}^{i}$ and,.., and $z_{\mathrm{n}}(t)$ is $M_{n}^{i}$

$$
\text { THEN } u(t)=-C A_{i} x-\frac{1+\lambda}{1-\lambda} \hat{d}_{i}(t, x) \operatorname{sgn}(S)
$$

The global fuzzy VSC and the adaptation laws are designed as

$$
\begin{aligned}
& u(t)=-\sum_{i=1}^{r} h_{i}(z)\left[C A_{i} x+\frac{1+\lambda}{1-\lambda} \hat{d}_{i}(t, x) \operatorname{sgn}(S)\right] \\
& \left\{\begin{array}{l}
\hat{d}_{i}(t, x)=d_{1 i}+d_{2 i}\|S\|+\frac{\lambda}{1+\lambda}\left\|C A_{i} x\right\|+\sum_{k=0}^{1} \hat{r}_{k}\|x\|^{k} \\
\dot{\hat{r}}_{0}=\gamma_{0}(1+\lambda)\|S\| \\
\dot{\hat{r}}_{1}=\gamma_{1}(1+\lambda)\|S\| \cdot\|x\|
\end{array}\right.
\end{aligned}
$$

where the parameters $d_{1 i}>0, d_{2 i}>0$, the adaptation rate $\gamma_{k}>0$, and the constant $\lambda=\sqrt{r}\|C \bar{B}\| \leq \mu\|\bar{B}\| \leq 1$ 。

The following theorem gives the stability analysis when the controller (30) is enforced on the system (4) and the reachability of the specified sliding surface $s(t)=0$ can be obtained.

Theorem 2: For the uncertain fuzzy systems (4) with the switching function (5), $Q$, $\alpha, \beta, \mu$ is the feasible solution of LMIs (6), (7) and (8). Then it can be shown that the state trajectories of the system (4) will be driven onto the switching surface $S(t)=0$ and asymptotically converge to zero by the adaptive VSC law in (29) and (30).

Proof: For purpose of design integrity, a simple stability analysis based on Lyapunov direct method is carried out. Define the Lyapunov function candidate

$$
V_{2}(t)=\frac{1}{2} S^{T} S+\sum_{k=0}^{1} \frac{1}{2 \gamma_{k}} \tilde{r}_{k}^{2}
$$

where the estimation error $\tilde{r}_{k}=\hat{r}_{k}-r_{k}$. By differentiating the function $V_{2}$, we obtain

$$
\dot{V}_{2}(t)=S^{T} \dot{S}+\sum_{k=0}^{1} \frac{\tilde{r}_{k}}{\gamma_{k}}
$$


Noting that

$$
\dot{S}=C \dot{x}=\sum_{i=1}^{r} h_{i}(z) C A_{i} x+[I+C \bar{B} \bar{H}(z) \bar{I}](u+g)
$$

Then, it follows from (4) that $\|C \bar{B} \bar{H}(z) \bar{I}\| \leq \sqrt{r}\|C \bar{B}\|=\lambda<1$, and we have

$$
\begin{aligned}
S \dot{S} & =S^{T} \sum_{i=1}^{r} h_{i}(z) C A_{i} x+S^{T}[I+C \bar{B} \bar{H}(z) \bar{I}] \cdot[u+g] \\
& \leq \sum_{i=1}^{r} h_{i}(z) S^{T} C A_{i} x+S^{T} u+[\lambda\|u\|+(1+\lambda)\|g\|] \cdot\|S\|
\end{aligned}
$$

By substituting the controller (29) into (34), we have

$$
\begin{aligned}
S \dot{S} & \leq \sum_{i=1}^{r} h_{i}(z) S^{T} C A_{i} x+S^{T}\left\{-\sum_{i=1}^{r} h_{i}\left[C A_{i} x+\bar{\lambda} \hat{d}_{i} \operatorname{sgn}(S)\right]\right\}+\lambda\left[\sum_{i=1}^{r} h_{i}\left\|C A_{i} x\right\|+\sum_{i=1}^{r} h_{i} \bar{\lambda}\left\|\hat{d}_{i}\right\|\right]\|S\|+(1+\lambda)\|g\|\|S\| \\
& =-\sum_{i=1}^{r} h_{i}(z)\left[\bar{\lambda} \hat{d}_{i} \cdot\|S\|-\bar{\lambda} \lambda\|\delta \cdot S\|\right]+\sum_{i=1}^{r} h_{i}(z) \lambda\left\|C A_{i} x\right\| \cdot\|S\|+(1+\lambda)\|g\| \cdot\|S\|
\end{aligned}
$$

where $\bar{\lambda}=\frac{1+\lambda}{1-\lambda}$. By substituting the adaptive laws (30) into (35), the simplified expression of (35) can be obtained as

$$
\begin{aligned}
S \dot{S} & =-(1+\lambda)\|S\|\left\{d_{1 i}+d_{2 i}\|S\|+\frac{\lambda}{1+\lambda}\left\|C A_{i} x\right\|+\sum_{k=0}^{1} \hat{r}_{k}\|x\|^{k}\right\}+\sum_{i=1}^{r} h_{i}(z) \lambda\left\|C A_{i} x\right\|\|S\|+(1+\lambda)\|g\|\|S\| \\
& =-(1+\lambda) \sum_{i=1}^{r} h_{i}(z)\left\{d_{1 i}\|S\|+d_{2 i}\|S\|^{2}\right\}-(1+\lambda)\|S\| \sum_{k=0}^{1} \tilde{r}_{k}\|x\|^{k}
\end{aligned}
$$

Thus, $\quad \dot{V} \leq-(1+\lambda) \sum_{i=1}^{r} h_{i}(z)\left\{d_{1 i}\|S\|+d_{2 i}\|S\|^{2}\right\}$. Noticing that $0<\lambda<1$, the derivative $\dot{V}<0$ when $S \neq 0$, which implies that under the controller (29) and (30) the reachability of the specified switching surface is guaranteed, and the trajectories of the fuzzy uncertain system (2) are globally driven onto the specified switching surface $S=0$. Moreover, it is seen that the estimation error $\tilde{r}_{k}$ will converge to zero.

\section{Numerical Simulation}

To show the effectiveness of the proposed controller design techniques, the inverted pendulum with parametric uncertainties, which is taken from $\mathrm{Wu}$ and Juang (2008), is formulated for simulation. The control objective is to drive its state trajectories to the origin. The equations of motion for the inverted pendulum device are 


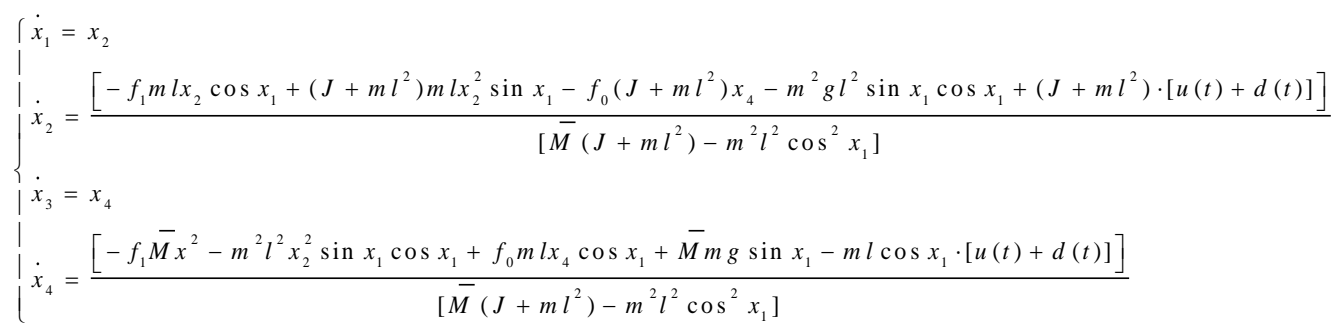

where $x_{1}$ denotes the angle ( $\left.\mathrm{rad}\right)$ of the pendulum from the vertical, $x_{2}$ is the angular velocity $(\mathrm{rad} / \mathrm{s}), x_{3}$ is the displacement $(\mathrm{m})$ of the cart, and $x_{4}$ is the velocity of the cart. $g=9.8 \mathrm{~m} / \mathrm{s}^{2}$ is the gravity constant, $m$ is the mass $(\mathrm{kg})$ of the pendulum, $M$ is the mass $(\mathrm{kg})$ of the Cart, $f_{0}$ is the friction factor $(\mathrm{N} / \mathrm{m} / \mathrm{s})$ of the cart, $f_{1}$ is the friction factor $(\mathrm{N} / \mathrm{rad} / \mathrm{s})$ of the pendulum, $l$ is the length $(\mathrm{m})$ from the center of the mass of the pendulum to the shaft axis, $J$ is the moment of inertia $(\mathrm{kg} . \mathrm{m} 2)$ of the pendulum round its center of mass, and $u$ is the force $(\mathrm{N})$ applied to the cart. The model parameters are given as: $\bar{M}=M+m, M=1.3282 \mathrm{~kg}, m=0.22 \mathrm{~kg}$, $f_{0}=22.915 \mathrm{~N} / \mathrm{m} / \mathrm{s}, \quad f_{1}=0.007056 \mathrm{~N} / \mathrm{rad} / \mathrm{s}, l=0.304 \mathrm{~m}, \mathrm{~J}=0.004963 \mathrm{~kg} . \mathrm{m} 2$ in the numerical simulation. It's assumed that $d(t)$ is bounded by $|d(t)| \leq \rho_{0}+\rho_{1}\|x\|$, where $\rho_{0}, \rho_{1}$ are unknown parameters.

The fuzzy model of system is described as the following two rules:

Plant rule 2: IF $\quad x_{1}$ is $M_{1}{ }_{1}^{1}$, THEN $\dot{x}=A_{1} x+B_{1}[u+g(t, x)]$

Plant rule 2: IF $\quad x_{1}$ is $M_{1}^{2}$, THEN $\dot{x}=A_{2} x+B_{2}[u+g(t, x)]$

$$
\text { where the model parameters }
$$
are

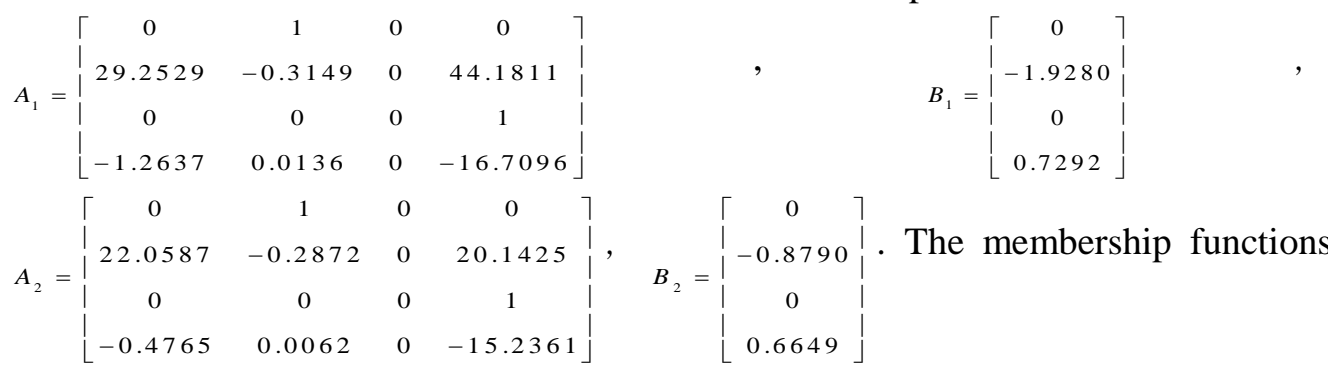
are selected as $M_{1}\left(x_{1}(t)\right)=\left[1-1 /\left(1+e^{-7\left(x_{1}-\pi / 24\right)}\right)\right] /\left(1+e^{-7\left(x_{1}+\pi / 24\right)}\right)$, $M_{2}\left(x_{1}(t)\right)=1-M_{1}\left(x_{1}(t)\right)$. Due to $B_{2} \neq B_{1}$, the stabilization result (Zheng et al., 2002) is invalid. The perturbation is set to be $g(t, x)=[0.1 \sin t \quad 0.05 \sin t]^{\mathrm{T}}$, the initial states $x(0)=[\pi / 3,0, \pi / 5,0]$. To assess the effectiveness of our fuzzy controller, we apply the controller to the original system (18) with nonzero $d(t)$. We choose the adaptation parameters $d_{1 i}=d_{2 i}=0.2, \gamma_{k}=0.001$.

Via LMI optimization with (19), we obtain the following solution matrices

$$
\begin{aligned}
& \boldsymbol{Q}=\left[\begin{array}{cccc}
0.0404 & -0.1790 & 0.0063 & -0.0268 \\
-0.1790 & 406.0261 & -0.0277 & -201.1424 \\
0.0063 & -0.0277 & 505.4098 & -0.0052 \\
-0.0268 & -201.1424 & -0.0052 & 99.9755
\end{array}\right], \alpha=1904.5, \quad \beta=1010.8, \\
& \mu=0.6951 \text {, }
\end{aligned}
$$

Hence, the switching surface in (5) can be obtained as 
$S(t)=\left[\begin{array}{llll}-86.4417 & -15.1532 & 0 & -29.0767\end{array}\right] x(t)$.

The simulation results are given in Figures 1-4. It is seen that the reachability of the sliding motion can be guaranteed. The system enters sliding-mode motion after about $t=0.8$ second. From Figure 1, one can see that the system states converge to zero fast, furthermore, the simulation results also show that our present design effectively attenuates the effect of both parameter uncertainties and external disturbances. Figure 3 and Figure 4 give the trajectory of the estimation of $r_{0}$ and $r_{1}$, respectively. In Figure 5, the control effort is shown and approaches to be stable after a short-term adjustment in the initial stage.

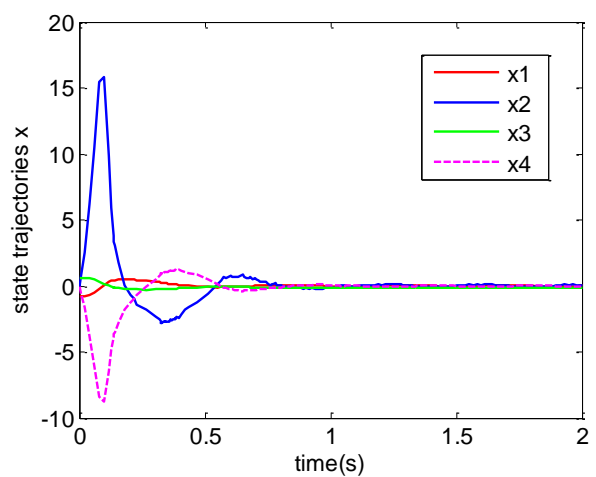

Figure 1. Trajectories of States $x 1, x 2, x 3, x 4$

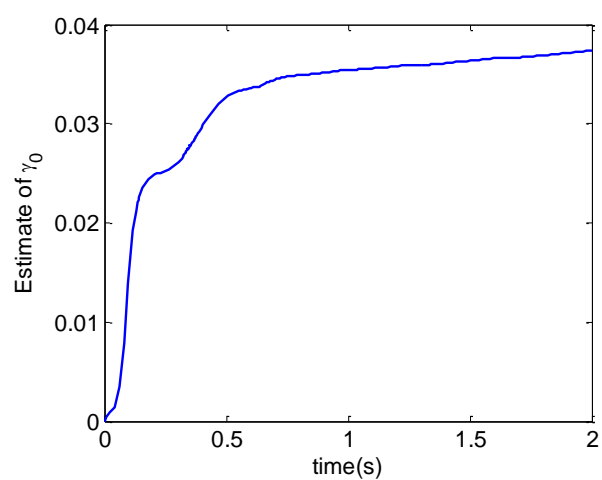

Figure 3. Trajectory of the Estimation of $r_{0}$

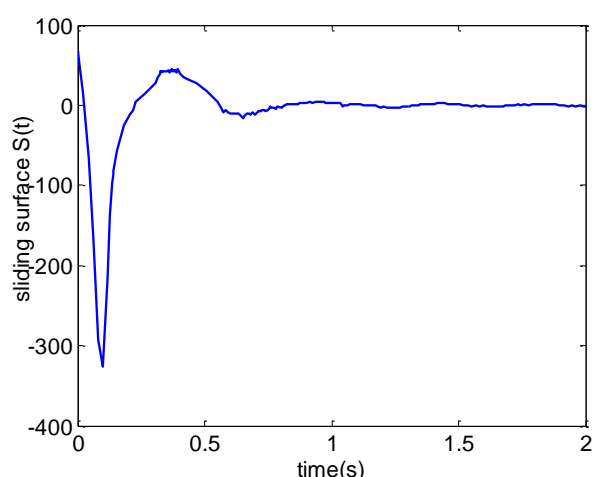

Figure 2. Trajectory of Switching Surface $S(t)$

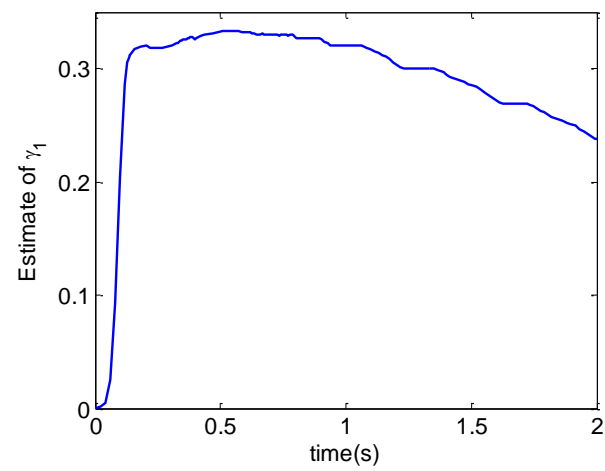

Figure 4. Trajectory of the Estimation of $r_{1}$

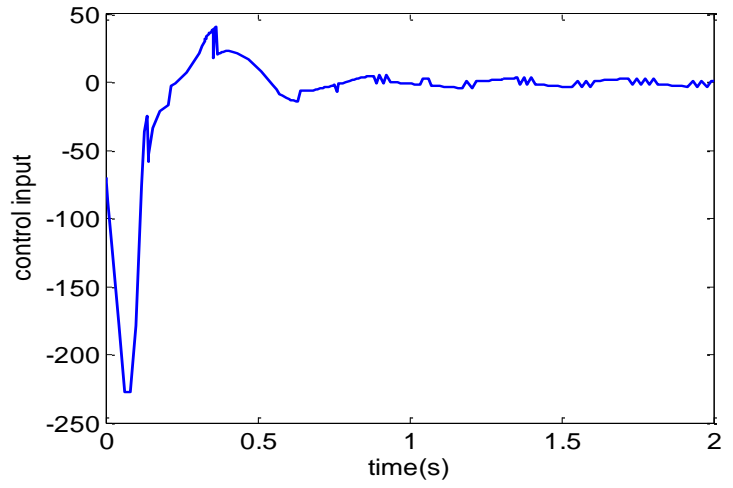

Figure 5. Control Effort $u(t)$ 


\section{Conclusion}

The T-S fuzzy model has been proven to be a very good representation for complex nonlinear systems. This paper has generalized the $\mathrm{T}-\mathrm{S}$ fuzzy model to represent a class of nonlinear systems which includes parameter uncertainties or external disturbances. A novel adaptive VSC control scheme has been proposed for the uncertain model, which relaxes the restrictive assumption that the input matrices of the local sub-models are identical and needs no information of uncertainties. The local VSC controller comprises of a state-feedback control term and an adaptive switching-feedback control term, which aims to estimate the upper bound of uncertainties on-line. The overall fuzzy VSC controller of the system is achieved by fuzzy blending of the local VSC controller. Via LMI optimization technique, the existence condition of linear sliding surfaces guaranteeing asymptotic stability of the reduced-order equivalent sliding mode dynamics is derived as well as the stability analysis by the Lyapunov function method. Finally, a numerical design example is illustrated in order to show the effectiveness of our scheme. It should be noted that the LMI existence condition (6)-(8) in this scheme may be more or less conservative, and in the future the research on a less conservative existence condition is to be planned.

\section{Acknowledgements}

The authors are grateful to the anonymous reviewers and to the support of the National Natural Science Foundation of China (61203019), the Key Project of Chinese Ministry of Education (No.212122) and the Natural Science Foundation of Hunan Provincial (No.13JJ9019).

\section{References}

[1] T. Takagi and M. Sugeno, "Fuzzy identification of systems and its applications to modeling and control", IEEE Transaction on System, Man, Cybernetic, vol. 15, no. 1, (1985), pp. 116-132.

[2] G. Feng, "A survey on analysis and design of model-based fuzzy control systems", IEEE Transaction on Fuzzy System, vol. 14, no. 5, (2006), pp. 676-697.

[3] K. Tanaka, T. Ikeda and H. O. Wang, "Fuzzy regulators and fuzzy observers: Relaxed stability conditions and LMI-based designs", IEEE Transactions on Fuzzy Systems, vol. 6, no. 2, (1998), pp. $250-265$.

[4] X. Z. Zhang and Y. N. Wang, "Fuzzy variable structure control based on a Takagi-Sugeno model for permanent magnet synchronous motors", ProcIME, Part I: Journal of Systems and Control Engineering, vol. 223, no. 6, (2009), pp. 773-783.

[5] A. H. Besheera, H. M. Emarab and M. M. A. Azizb, "Wind energy conversion system regulation via LMI fuzzy pole cluster approach", Electric Power Systems Research, vol. 79, no. 4, (2009), pp. 531-538.

[6] S. Aouaouda, M. Chadli and M. Tarek Khadir, "Robust fault tolerant tracking controller design for unknown inputs $\mathrm{T}-\mathrm{S}$ models with un-measurable premise variables", Journal of Process Control, vol. 22, no. 5, (2012), pp. 861-872.

[7] Y. W. Liang, S. D. Xu and D. C. Liaw, "A study of T-S model-based SMC scheme with application to robot control", IEEE Transactions on Industrial Electronics, vol. 55, no. 11, (2008), pp. 3964 3971.

[8] E. G. Tian and C. Peng, "Delay dependent stability analysis and synthesis of uncertain T-S fuzzy systems with time-varying delay", Fuzzy Sets System, vol. 157, no. 4, (2006), pp. 544-559.

[9] H. N. Wu, "Robust $\mathrm{H}_{2}$ fuzzy output feedback control for discrete-time nonlinear systems with parametric uncertainties", International Journal of Approximate Reasoning, vol. 46, no. 1, (2007), pp. 151-165.

[10] Y. C. Lin and J. C. Lo, "Robust mixed $\mathrm{H} 2 / \mathrm{H} \infty$ filtering for discrete-time delay fuzzy systems", International Journal of System Science, vol. 36, no. 15, (2005), pp. 993-1006.

[9] C. Edwards and S. K. Spurgeon, "Sliding mode control: Theory and application. Taylor \& Francis Ltd.", London, (1998).

[10] Z. Zuo and Y. N. Wang, "Robust stability and stabilisation for nonlinear uncertain time-delay systems via fuzzy control approach”, IET Control Theory, vol. 1, no. 1, (2007), pp. 422-429. 
[11] F. Zheng, Q. G. Wang and T. H. Lee, "Output tracking control of MIMO fuzzy nonlinear systems using variable structure control approach", IEEE Transaction on Fuzzy System, vol. 10, no. 6, (2002), pp. 686-697.

[13] W. J. Cao and J. X. Xu, "Nonlinear integral-type sliding surface for both matched and unmatched uncertain systems", IEEE Transaction on Automatic Control, 2004, vol. 49, no. 8, (2010), pp. 1355 1360.

[14] P. Gahinet, A. Nemirovski, A. J. Laub and M. Chilali, "LMI Control Toolbox", The MathWorks, Natick, Massachusetts (1995).

[15] H. H. Choi, "Robust stabilization of uncertain fuzzy systems using variable structure system approach", IEEE Transactions on Fuzzy Systems, vol. 16, no. 3, (2010), pp. 715-724.

[16] X. Z. Zhang, Y. N. Wang and X. F. Yuan, "Hळ robust T-S fuzzy design for uncertain nonlinear systems with state delays based on sliding-mode control", International Journal of Computers, Communications \& Control, vol. 5, no. 4, (2010), pp. 592-602.

[17] T. Z. Wu and Y. T. Juang, "Design of variable structure control for fuzzy nonlinear systems", Expert Systems with Applications, vol. 35, no.3, (2008), pp. 1496-1503.

[18] H. K. Khalil, "Nonlinear systems", (3rd edition), Prentice-Hall Inc., New Jersey, (2002).

\section{Author}

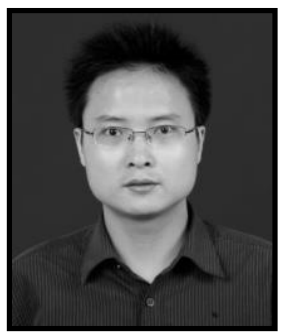

Xizheng Zhang, he received the B.S. degree in electrical engineering, the M.S. degree in circuits and systems and control engineering in 2000, 2003 and 2010, respectively, from Hunan University, China, He is also with the School of Computer and Communication, Hunan Institute of Engineering, China. His research interests include control theory and its application. 